\title{
Wireless recording of the calls of Rousettus aegyptiacus and their reproduction using electrostatic transducers
}

\author{
S M Whiteley ${ }^{1}$, D A Waters ${ }^{2}$, G Hayward ${ }^{1}$, S G Pierce ${ }^{1}$ and I Farr ${ }^{2}$ \\ ${ }^{1}$ Centre for Ultrasonic Engineering, EEE Department, University of Strathclyde, Glasgow, UK, G1 1XW \\ ${ }^{2}$ Institute of Integrative and Comparative Biology, University of Leeds, Leeds, LS2 9JT, UK. \\ E-mail: swhiteley@eee.strath.ac.uk
}

\begin{abstract}
Bats are capable of imaging their surroundings in great detail using echolocation. To apply similar methods to human engineering systems requires the capability to measure and recreate the signals used, and to understand the processing applied to returning echoes. In this work, the emitted and reflected echolocation signals of Rousettus aegyptiacus are recorded while the bat is in flight, using a wireless sensor mounted on the bat. The sensor is designed to replicate the acoustic gain control which bats are known to use, applying a gain to returning echoes that is dependent on the incurred time delay. Employing this technique allows emitted and reflected echolocation calls, which have a wide dynamic range, to be recorded. The recorded echoes demonstrate the complexity of environment reconstruction using echolocation. The sensor is also used to make accurate recordings of the emitted calls, and these calls are recreated in the laboratory using custom-built wideband electrostatic transducers, allied with a spectral equalisation technique. This technique is further demonstrated by recreating multi-harmonic bioinspired FM chirps. The ability to record and accurately synthesize echolocation calls enables the exploitation of biological signals in human engineering systems for sonar, materials characterisation and imaging.
\end{abstract}

Keywords: Rousettus aegyptiacus, echolocation, telemetry, electrostatic transducers, spectral inversion

PACS Codes. 43.80.Ev, 43.80.Ka, 43.38.Bs

\section{Introduction}

Since Donald Griffin and his colleagues (Griffin, 1944) first demonstrated that bats use echolocation for navigation and predation, this process has been the subject of much research. The ability of bats to discriminate the absolute range of an acoustic target (Simmons, 1973), range jitter (Simmons, 1979; Moss and Schnitzler, 1989) or angular direction (Simmons et al., 1983; Lawrence and Simmons, 1982) has been well established. The methods bats use to resolve target range have been the subject of much research and discussion (e.g. Simmons, 1973; Simmons, 1979; Moss and Schnitzler, 1989). Jitter discrimination tests suggest that bats perform a correlation of emitted and reflected waveforms, and that they may be capable of using the fine detail of this correlation function in the time domain (Simmons et al., 1990). Since this process requires the bat to store a representation of the emitted signal, a useful step in providing an improved understanding of bat echolocation is to obtain an accurate representation of this signal.

Conventional recording of bat calls has been performed using a static or hand-held microphone, coupled to a recording medium. This method suffers from inherent problems, summarized by Waters and Jones (1995), such as the frequency-dependence of both the attenuation in air and the emitted beam pattern. These effects make accurate recording of calls from a flying bat difficult to achieve, and a number of techniques have been employed to reduce their impact. Laboratory-based recordings have been performed either by holding the bat stationary (Hartley and Suthers, 1987), or in a pendulum (e.g. Henson et al., 1987), to enable recordings to be made under known conditions. This can lead to uncharacteristic recordings, since flying bats energetically couple calls whilst in flight (Speakman and Racey, 1991; Lancaster et al., 1995). Recently, both field and laboratory studies have used methods to locate a free-flying bat in space at the time of a call emission, in relation to a high-fidelity recording microphone, thereby allowing compensation for atmospheric attenuation. The methods employed use either an array of microphones (e.g. Surlykke and Kalko, 2008) or stereo videogrammetry (Ghose and Moss, 2003; Holderied et al., 2005). 
None of these techniques, however, provide any information on either the emitted signal as heard by the bat (which may provide a template for subsequent cross-correlation) or on the signal as received by the bat from a target; the difference between these signals being the basis for the echolocation process. One method which can give an insight into these signals uses a wireless microphone sensor mounted on a bat, to allow calls to be detected as it echolocates in flight in a captive environment. Studies using this method (e.g. Lancaster et al., 1992; Hiryu et al., 2005) have successfully demonstrated its usefulness in recording the calls of both CF (constant frequency) and FM (frequency modulated) bats in flight. Indeed, the system developed by Hiryu et al. $(2007 ; 2008)$ was very lightweight $(0.6 \mathrm{~g})$ and has been used successfully with a number of species flying in captivity. Given the fixed small distance between the bat's mouth and microphone, this avoids many of the problems associated with atmospheric attenuation in recording an accurate echolocation call, in addition to providing a reference signal for cross correlation.

These developments have shed much light on the signals used by bats during echolocation. However, the systems reported to date have limited dynamic range, and the lack of detailed calibration makes accurate signal quantification difficult. The work reported here has two specific aims: to provide tools to further investigate the echolocation process; and to enable the potential for biomimetic bat sonar to be employed in human engineering systems. A wireless sensor is described that was mounted on a bat whilst it flew in a captive environment. The sensor can be configured either to capture high-fidelity recordings of the emitted signal, or to emphasize recording of the returning echoes to mimic the natural acoustic gain control system of the bat. This is achieved by dynamically controlling the level of amplification through the sensor. The sensor was accurately calibrated to gain an insight into how measured signals were affected during the recording process.

A second aspect of the work involved the accurate replication of wideband bat calls within the laboratory environment. Calibration of the sensor system revealed severe limitations in current transducer technology with respect to sensitivity across the bandwidth of the bat spectral range (i.e. $15 \mathrm{kHz}-200 \mathrm{kHz}$ ). Through reciprocity, such difficulties would also be anticipated in transmission. Accurate replication of a wide range of bat echolocation signals will facilitate the study of such signals and the associated binaural processing for target classification and range discrimination. This in turn could pave the way for an extensive range of engineering applications, including distance measurement, robotic navigation, underwater navigation and materials characterization. Of particular interest to the authors is application of bat signals in ranging, to enable accurate location and positioning of robots (Friedrich et al., 2009).

\section{Wireless sensor design}

A central aim of the current work was to create a sensor system capable of detecting both the emitted signal and the echoes bats use in echolocation, providing an insight into the signals that bats decipher to form an image of their environment. This was a significant challenge, given the bandwidth and dynamic range of many echolocation signals. Emitted signals have been estimated with a peak pressure in excess of $140 \mathrm{~dB}$ peSPL in the field (Surlykke and Kalko, 2008), with audiograms of bats indicating a hearing threshold in the region of 0dB (e.g. Neuweiler et al., 1984; Koay et al., 2003). While this is a hearing threshold (i.e. the level at which a bat becomes aware of the sound) rather than the level at which that sound becomes useful for echolocation, bats are certainly capable of processing signals over a significant dynamic range.

The intended test subject for the wireless sensor was the fruit bat Rousettus aegyptiacus, a member of the only genus within the suborder megachiroptera that is known to use echolocation. The calls generally consist of pairs of 'clicks' produced with the tongue. While these signals may appear less complex than the signals produced by microchiropterans, one study on $R$. aegyptiacus (Waters and Vollrath, 2003) indicates that its performance in wire avoidance tests is comparable to such species. $R$. aegyptiacus is also fairly large and robust, which makes it an ideal candidate for flight experiments with a wireless microphone sensor. In terms of mass requirements, long-term studies have indicated load carrying should not impose more than a 5\% increase in mass to avoid undue stress on the subject (Aldridge and Brigham, 1988). However, for short term laboratory based studies loads of up to $10 \%$ of the bat body mass should not impose any undue strain, since gravid bats often reach a $20 \%-30 \%$ increase in body mass in the latter stages of pregnancy (Korine et al., 2004). The target frequency range for the sensor was $20 \mathrm{kHz}-200 \mathrm{kHz}$ in order to allow future work with FM and CF bat species. 
Wireless sensors reported in the literature consist of a microphone coupled via an amplifier to a suitable oscillator for radio frequency (RF) transmission. However, given that the target bat species was larger than the species used in previous studies, there was some latitude in size and weight constraints. Furthermore, a fixedgain amplifier would not produce a sensor capable of operating across a wide enough dynamic range. Research has shown that some bats, such as Tadarida brasiliensis mexicana (Henson, 1965), Myotis lucifugus (Jen and Suga, 1975), and Eptesicus fuscus (Kick and Simmons, 1984), have evolved a mechanism that helps them to overcome the dynamic range between emitted and reflected signals. By contraction of the middle ear muscles just prior to emission of the echolocation call, a temporary reduction in hearing sensitivity is achieved for the period of call emission. This sensitivity is then gradually restored as the muscles relax post emission, causing sensitivity to increase as the propagation loss incurred by echoes increases (Kick and Simmons, 1984; Simmons et al., 1992). Hence, the bat employs gain control in order to regulate the perceived loudness of the returning echoes, thereby increasing the dynamic range of signals it can process. Through use of a Variable Gain Amplifier (VGA) and suitable control circuitry, this can be recreated electronically, allowing echoes to be subjected to an increasing level of amplification, based on echo delay.

The main components of the sensor system were a microphone, instrumentation amplifier, VGA and voltage controlled oscillator, with the latter used to generate FM signals for wireless transmission. In addition, the system included timing circuitry for control of the VGA, a battery and antenna. The microphone selected for use was the FG3329 electret microphone (Knowles, IL, USA), the same model as used by Hiryu et al. (2005; 2007; 2008). Manufacturer data indicates that device sensitivity decreases beyond $20 \mathrm{kHz}$, but should still be usable above $100 \mathrm{kHz}$ making it suitable for use in this system. In the course of the investigation, it was shown that the response of this microphone was seriously impaired at frequencies above $120 \mathrm{kHz}$, as discussed in section 3. The microphone was coupled to an AD623 instrumentation amplifier (Analog Devices, MA, USA) to provide an initial gain stage and common-mode noise immunity. Variable gain was then provided by the AD8330 VGA (Analog Devices), with the amplified signal applied to a MAX2606 (Maxim, CA, USA) voltage controlled oscillator that can generate FM radio signals across a wide range of frequencies, including the commercial $(88 \mathrm{MHz}-108 \mathrm{MHz}) \mathrm{FM}$ band. Control of the gain circuitry was provided by a timer circuit based on the TLC555 (Texas Instruments, TX, USA) device. Finally, power was provided by a 3.6V nickel-metal hydride (Ni-MH) rechargeable 3/V15H battery (Varta Microbattery, Ellwangen, Germany). This enabled the sensor to be powered for 20 minutes between charging, which was sufficient for bat flight tests in captivity. A wire of $19 \mathrm{~cm}$ in length (approximately $\lambda / 16$ for radio waves at a frequency of $100 \mathrm{MHz}$ ) was attached to the sensor as a whip antenna. With this configuration, reliable reception of signals from the sensor was possible at distances up to $10 \mathrm{~m}$ in cluttered laboratory space.

In variable-gain mode, the sensor was initially set to its lowest gain level. When an emitted echolocation call was detected, a charging circuit was configured to apply a rising voltage to the gain-control pins of the VGA. The time period over which this voltage rose was defined through selection of the appropriate resistor and capacitor combination. This allowed the sensor to be configured for operation over a given timespan, corresponding to a given range. After this time period, the sensor was reset to its lowest gain state in preparation for the next emitted call. In this way, echo signals were subjected to additional gain dependent on their time of arrival, as is the case with the gain control mechanism seen in the auditory pathway of bats. A measured gain variation of $67 \mathrm{~dB}$ was achieved, that was shown to significantly improve the detection of echoes returned to the bat. It was also possible to operate the sensor in static gain mode, by fixing the voltage applied to the VGA.

The sensor weighed $6.8 \mathrm{~g}$, representing a maximum of $8 \%$ of the body mass of the bat, which ranged from $85 \mathrm{~g}$ to $139 \mathrm{~g}$. The majority of the sensor mass was attributable to the battery $(4.0 \mathrm{~g})$. This battery was necessary since the sensor required a current of approximately $25 \mathrm{~mA}$, mostly attributable to the variable gain circuitry. The prototype wireless sensor is pictured in Figure 1, attached to a $R$. aegyptiacus individual. The transmitted FM signal was received by a 70FT930 FM tuner (Philips, Eindhoven, The Netherlands) used in one of two ways: firstly without modification, but extracting the demodulated signal prior to baseband filtering, allowing reception of signals up to approximately $100 \mathrm{kHz}$; and secondly by using a custom-built phase locked loop 
(PLL) circuit to demodulate the $10.7 \mathrm{MHz}$ Intermediate Frequency (IF) signal generated by the mixer in the tuner. The PLL was required to facilitate demodulation of higher frequency signals at up to $200 \mathrm{kHz}$.

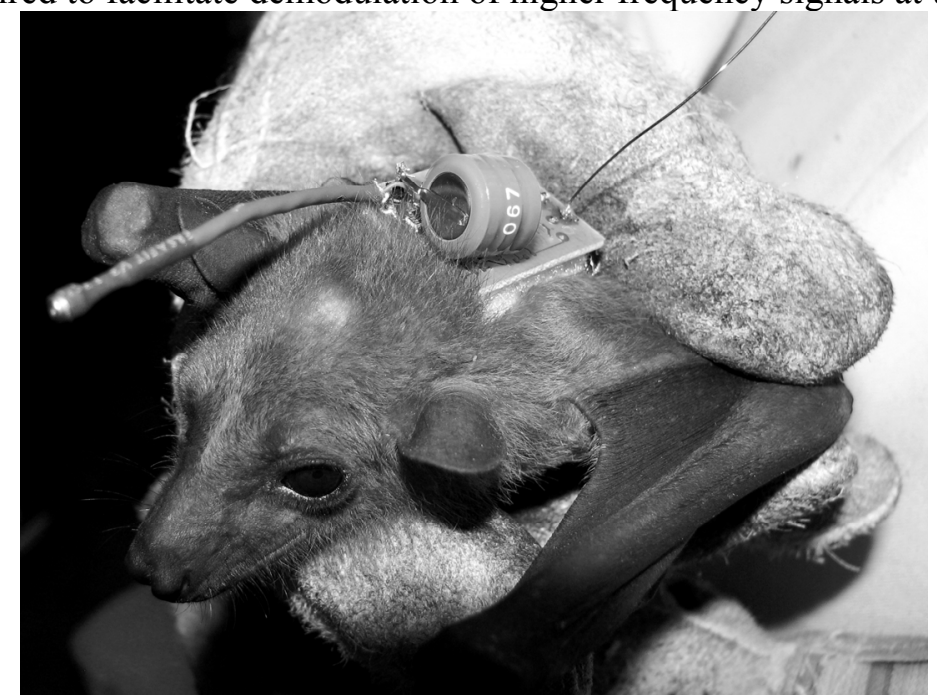

Figure 1. R. aegyptiacus with wireless sensor mounted dorsally, between the shoulder blades. The microphone, battery and rear-mounted whip antenna can be seen.

\section{System Calibration}

To gain an understanding of the influence that the sensor system had on the signals it recorded, its frequency response was characterized using the standard substitution method. The transmission frequency response of a custom-built, $30 \mathrm{~mm}$ diameter, wideband electrostatic transducer (described in section 6.1) was measured using a calibrated Type 4138 microphone coupled to a Nexus 2690 preamplifier (both Brüel \& Kjær, Nærum, Denmark). This was performed at a distance of $200 \mathrm{~mm}$, in the far field of the transducer for all frequencies. The wireless sensor system was then substituted for the Brüel \& Kjær (B\&K) microphone, with the FG3329 microphone positioned in place of the B\&K device, allowing the response of the sensor system to be calculated.

The response was measured using a series of Hanning-windowed 10-cycle toneburst signals, applied at $1 \mathrm{kHz}$ intervals across the frequency range of interest. The received signal was captured from a CS82G GaGe analogue to digital (A/D) converter card (Dynamic Signals, IL, USA). The process was automated using LabVIEW (National Instruments, TX, USA) to configure a 33120A (Agilent, CA, USA) Arbitrary Function Generator (AFG) and capture the received data from the A/D card. The sensor was configured in static gain mode, and transmitted signals were received using the FM tuner, both with and without the PLL demodulator as previously described. This resulted in the calculated frequency response shown in figure 2(a), with data being normalized. With the unmodified tuner, signals above $100 \mathrm{kHz}$ were not detectable, while the PLL demodulator extended this range up to approximately $150 \mathrm{kHz}$. It can be seen that the system response is seriously impaired at higher frequencies, with a steep roll-off above $120 \mathrm{kHz}$ that limits its use to frequencies below this value. The reason for this response was investigated by measuring the frequency response of each of the component parts of the sensor. The general low-pass nature of the response is produced by a combination of the microphone, oscillator and receiver electronics. The significant reduction in sensitivity above $120 \mathrm{kHz}$ was caused solely by the microphone, with a distinct null in its response at approximately $150 \mathrm{kHz}$. This response was not predicted by the manufacturer, and was investigated further. Finite-element analysis revealed that a resonant mode within the case of the microphone was responsible for the drop in sensitivity. This could not be removed without recourse to significant design modification, which was not straightforward due to the microphone design. To demonstrate that the remainder of the system was capable of operating over the target frequency range, an electronic test signal was injected into the instrumentation amplifier on the sensor, and the demodulated output from the tuner was measured. The response of this section is displayed in figure 2(b), where it can be seen that the PLL demodulator does indeed allow the system to operate over the intended frequency range. 

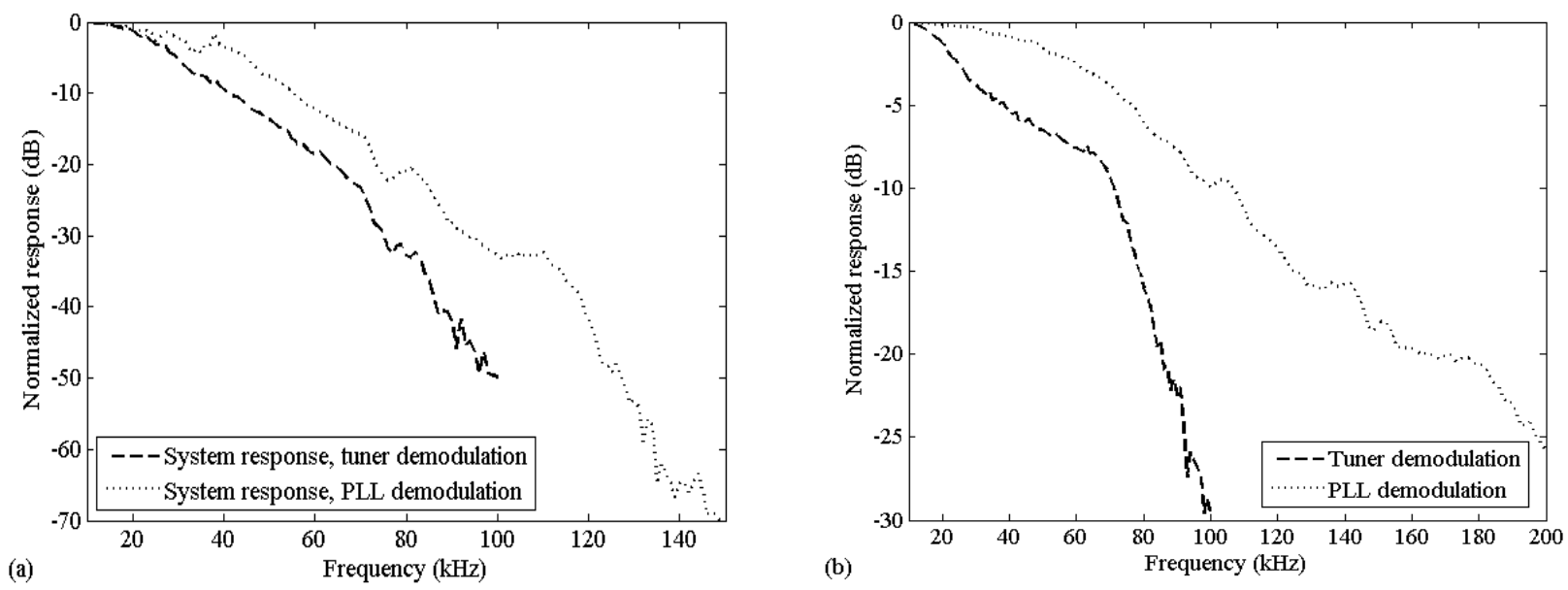

Figure 2. Calibration of the sensor system response using the substitution method. (a) Calculated response of system, using the in-built tuner demodulation and PLL demodulation. (b) Electronic system response, not including microphone.

\section{System Testing}

The calls of $R$. aegyptiacus consist of pairs of "clicks" produced by the tongue. This typically creates pulses of a few cycles in length, with a duration of approximately $250 \mu$ s and peak energy at approximately $19 \mathrm{kHz}$ (Waters and Vollrath, 2003 - includes averaged time-domain waveform, frequency spectrum and spectrogram). Most energy $(-6 \mathrm{~dB})$ is concentrated between $15 \mathrm{kHz}$ and $25 \mathrm{kHz}$, with little energy $(-15 \mathrm{~dB})$ below $15 \mathrm{kHz}$ or above $40 \mathrm{kHz}$. The peak pressure output is approximately $100 \mathrm{~dB}$ SPL. For this reason, testing of the sensor was carried out with a range of tonebursts at frequencies between $10 \mathrm{kHz}$ and $50 \mathrm{kHz}$ at a peak pressure of $100 \mathrm{~dB}$ SPL. A test was designed that enabled the fidelity of the sensor to be tested simultaneously with its variable gain response. This test used an air-coupled ultrasonic transducer to ensonify a steel target (a bar of cross section $20 \mathrm{~mm}$ ) at a distance of approximately $1.4 \mathrm{~m}$. The wireless sensor and B\&K microphone (for comparison purposes) were placed directly in the path of the acoustic energy between the transducer and the target, approximately $0.2 \mathrm{~m}$ from the transducer. In this way, the sensor and microphone were capable of detecting both the emitted signal, and the signal returning after reflection from the steel target. The signals detected by each system were captured using a digitizing oscilloscope.

Waveforms resulting from the test carried out with a $50 \mathrm{kHz}$ toneburst are displayed in figure 3. Figure 3(a) depicts the response from the B\&K microphone and wireless sensor to both emitted and reflected signals; the wireless sensor output is magnified 30 times, and both signals de level-shifted for clarity of display. The magnification was carried out digitally in post-processing, simply to allow visual comparison of the signals on the same scale. The variable gain performance is illustrated by the relative magnitudes of emitted and reflected signals from each detector. The drop in amplitude from transmitted to reflected signal is in the order of $30 \mathrm{~dB}$, as the B\&K microphone output demonstrates; this reduction is more than offset by the increased gain of the wireless sensor when the echo arrives. In this case the sensor was configured to have a gain variation of approximately $45 \mathrm{~dB}$ between its lower and upper limits, in order to preserve the clarity of the signals displayed in figure 3(a). Figure 3(b) shows the transmitted signal in more detail, illustrating the fidelity of the wireless sensor in comparison with the signal detected by the B\&K microphone. The magnification required of the wireless sensor signal ( $\times 180$ in this case, again done in post processing for visual comparison) is due to the sensor being in its default, low-gain state at this point. Figure 3(c) illustrates a comparison of the reflected signal recorded with the wireless sensor and B\&K microphone. It should be noted that no averaging of the resulting waveforms was performed. 

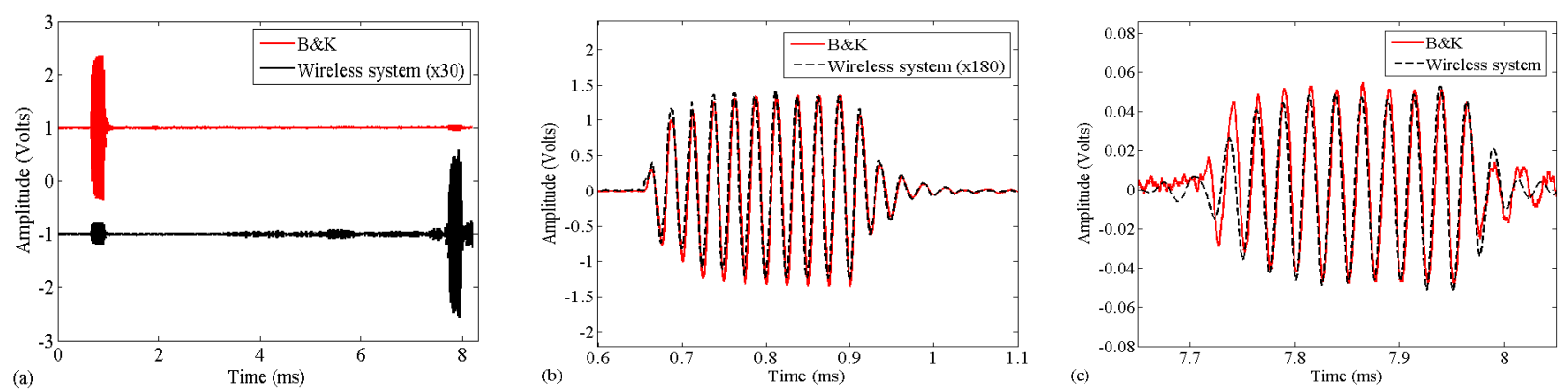

Figure 3. Signals from laboratory testing of the wireless sensor demonstrating dynamic gain performance and accuracy of the detected signal. Also shown are signals obtained with a calibrated B\&K Type 4138 microphone, for comparison. (a) Emitted and reflected signals (both dc level-shifted, with the wireless system signal amplitude multiplied by 30 for clarity). (b) Emitted signal (wireless system signal amplitude multiplied by 180 for comparison). (c) Signal reflected from target.

\section{Capturing echolocation signals from $R$. aegyptiacus in flight}

Six adult $R$. aegyptiacus were loaned from Tropical World, Leeds and maintained on a $12 \mathrm{~h}$ light, $12 \mathrm{~h}$ dark reversed photoperiod, in a cage continually provisioned with fruit and water. Experimental flights were conducted in a $14 \mathrm{~m} \times 2.5 \mathrm{~m} \times 1.5 \mathrm{~m}$ corridor situated adjacent to the room containing the cage. A mesh suspended at one end of the corridor served as an artificial roost, while a $100 \mathrm{~mm}$-diameter disc suspended from the ceiling half-way along the corridor provided an acoustic target. The sensor was attached to the bat using self-adhesive Velcro (a hook and loop fastener), with one piece attached dorsally between the shoulders of the bat, while the mating piece of Velcro was attached to the underside of the sensor. The sensor mounted on the bat can be seen in figure 1. Each bat performed up to 16 flights, each providing $3 \mathrm{~s}$ of acoustic data sampled at $400 \mathrm{kHz}$, as well as $3 \mathrm{~s}$ of high-speed video. In-depth analysis of these data will be the subject of further publications.

With $R$. aegyptiacus producing a double-click echolocation call on average every $86 \mathrm{~ms}$ (Waters and Vollrath, 2003), there was the potential for gathering large amounts of data on each $3 \mathrm{~s}$ flight recording. In practice, not all flights produced noise-free recordings - the corridor was not screened and as such was subject to varying electromagnetic interference - but well over 500 individual calls were captured, examples of which are presented in figures 4 and 5. Figure 4 depicts signals captured with the sensor configured to have fixed gain. This mode of operation is useful for capturing accurate representations of the emitted echolocation calls, with the best achievable signal to noise ratio (SNR), since the gain is set to an optimal level to capture these signals. The regular pattern of double clicks, characteristic of this species, can be clearly seen in figure 4(a), whilst figure 4(b) shows a representative example of a single echolocation call.
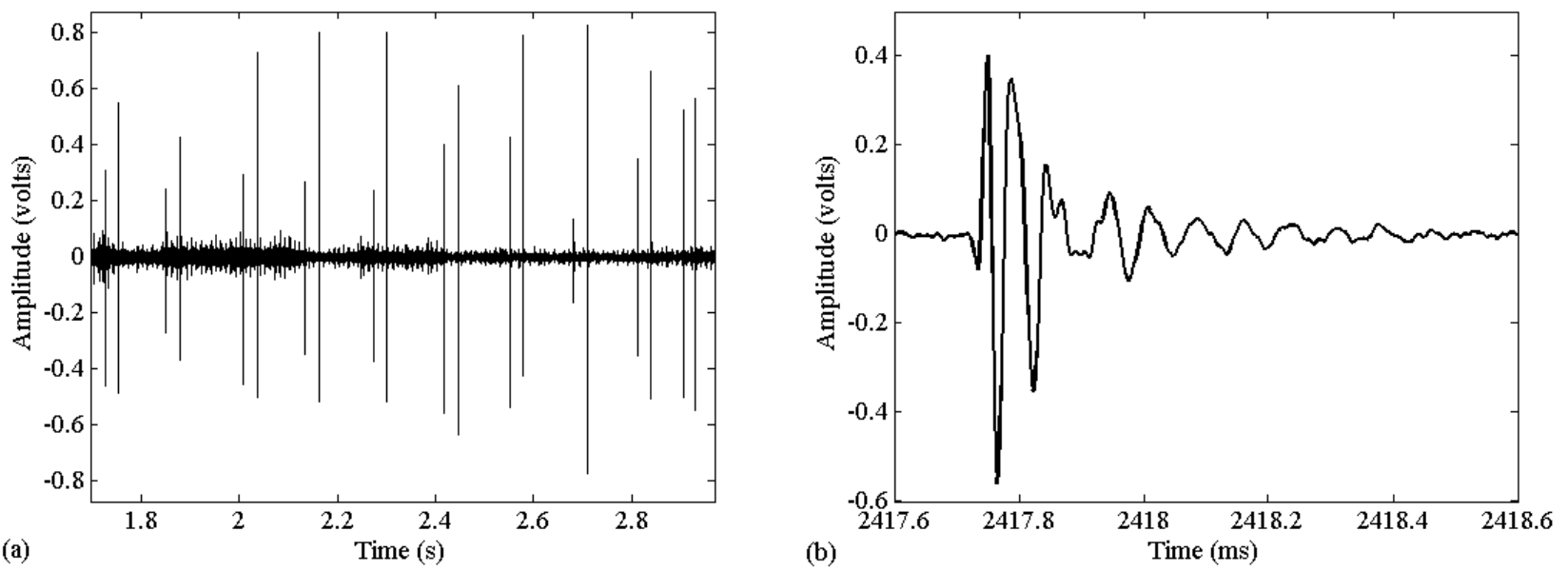

Figure 4. Echolocation signals captured using the wireless sensor in static gain mode on $R$. aegyptiacus whilst in flight. (a) Sequence of calls, showing "double-click" pattern. (b) Expanded image of individual call. 
By using the sensor in variable gain mode, the data illustrated in figure 5 was obtained. Figure 5(a) is provided for direct comparison with figure 4(a). These figures are not the same data recorded using static and variable gain. They simply have the same timespan and so allow comparison of the types of signals recorded using the sensor with either configuration. This illustrates the quantity of reflected signals returning to the bat recordable through use of variable gain. Given the range of hearing of $R$. aegyptiacus extends down to approximately $0 \mathrm{~dB}$ SPL (Koay et al., 1998), these signals would certainly be audible. Figure 5(b) depicts a single echolocation call and its associated echoes as recorded using variable gain. This shows reflected signals originating from objects in excess of $4 \mathrm{~m}$ from the bat - possibly from doorframes or light fittings. Also displayed in figure 5(b), on the same scale for comparison purposes, is a level-shifted trace captured using static gain. This illustrates the advantage of using variable gain to examine the reflected signals. It is immediately apparent, even in the relatively simple environment of a corridor, that the bat must decipher a complex combination of overlapping echoes from multiple objects and surfaces. Figure 5(c) displays a close-up version of the two furthest echoes detected by the sensor.
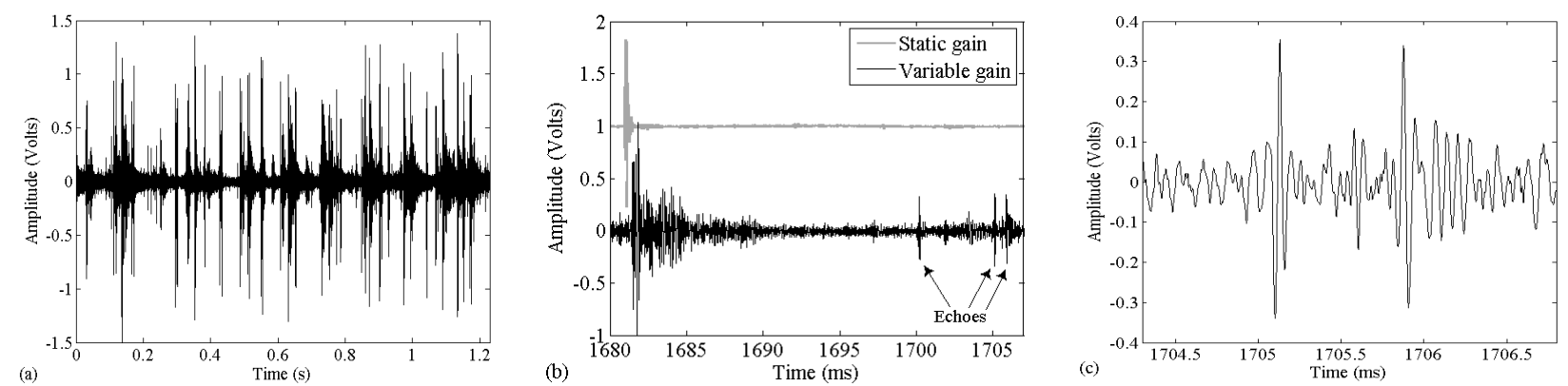

Figure 5. Echolocation signals captured using the wireless sensor in variable gain mode on $R$. aegyptiacus whilst in flight. (a) Sequence of calls, and echoes. (b) Single call and associated echoes, with de level-shifted version of a call recorded in static gain mode for comparison. (c) Close-up of echoes.

\section{Echolocation Call Replication}

Given the capability of bats to perceive their environment in great detail using echolocation, the use of similar methods would be advantageous in ranging and positioning applications. For example, an application that is of considerable interest to the authors is positioning of autonomous robotic vehicles used for Non-Destructive Evaluation (NDE). Enabling such a system to use biological, or biologically-inspired, acoustic signals could improve positional accuracy using correlation-based methods. In this respect, the wideband signals employed by $R$. aegyptiacus are attractive. Their relatively low frequency minimizes attenuation, while retaining sufficient bandwidth to utilize correlation and phase-detection techniques for accurate range discrimination.

\subsection{Air-coupled transducers}

As reviewed elsewhere (e.g. Manthey et al., 1992) there are a variety of transduction methods suitable for ultrasonic generation in air. However, current ultrasonic transducer technology has inherent limitations, both in terms of sensitivity and bandwidth. For example, piezoelectric devices are not well matched to the air environment and they are highly resonant with a narrow frequency bandwidth, especially when considered with a suitable matching layer (Kelly et al., 2004). Electrostatic transducers, comprising a thin membrane suspended above an electrically conducting backplate are more wideband and better matched to the air propagation channel (Schindel et al., 1995). However, whilst some of the more narrowband devices (Rafiq and Wykes, 1991) can operate in the frequency regime of $R$. aegyptiacus, wideband designs tend to struggle at these comparatively low frequencies, below $100 \mathrm{kHz}$ (Schindel et al., 1995). In this work, wideband air-coupled ultrasonic transducers were designed, and allied to a spectral inversion technique, as detailed in the following sections. For accurate correlation, knowledge is required of the acoustic signal that is actually transmitted; this signal depends on the response of the transducer and so may be considerably different from the driving electrical signal. It is for this reason that the spectral inversion process has been used.

Although the calls that $R$. aegyptiacus produces are mainly limited to less than $40 \mathrm{kHz}$ in frequency, it was an aim of the work reported here to enable the reproduction of the echolocation call of any bat. This included the 
more complex coded FM and CF-FM waveforms, as well as the clicks that $R$. aegyptiacus produces. This covers a frequency range from the audible to in excess of $200 \mathrm{kHz}$ (Fenton and Bell, 1981), representing a centre frequency in the region of $100 \mathrm{kHz}$ and a fractional bandwidth approaching $200 \%$. Bat call reproduction is a subject that is only sparsely and indirectly covered in the literature. This may be due to a lack of the hardware and tools to make it viable. Certainly the requirements and limitations are rigorous, since working with air as the load medium introduces a significant mechanical impedance mismatch with almost any wideband transduction material. Furthermore, in order to generate well-defined and predictable fields, pistonmode operation of the transducer is desirable across the frequency range of interest.

The electrostatic transducer designed and manufactured for this work consisted of a vitreous carbon backplate and $8 \mu \mathrm{m}$ Kapton transmitting membrane, with aluminum evaporated onto the front face, held in an aluminium case. The vitreous carbon was supplied polished smooth to within $50 \mathrm{~nm}$ in $30 \mathrm{~mm} \varnothing \times 5 \mathrm{~mm}$ pieces (HTW Hochtemperatur-Werkstoffe, Thierhaupton, Germany). The smooth finish of the backplate helps to promote a high-bandwidth response. The frequency response of this device was calculated from the impulse response of a pair of identical transducers arranged in a pitch-catch configuration. The transmitter was driven with a Panametrics 5052PR (Olympus Corporation, Tokyo, Japan) pulser unit, with the receiving transducer coupled to a Cooknell CA7 charge amplifier (Cooknell Electronics Ltd., Weymouth, UK). The resulting received signal was captured using a digitizing oscilloscope and is displayed in figure 6(a); the magnitude of the FFT (Fast Fourier Transform) of this signal is displayed in figure 6(b). This illustrates its relatively wideband response, with a peak output at $425 \mathrm{kHz}$ and a $6 \mathrm{~dB}$ pitch-catch bandwidth extending from $125 \mathrm{kHz}$ to $810 \mathrm{kHz}$, representing a fractional bandwidth of some $160 \%$. For recreating echolocation calls, however, the frequency range of interest extends to in excess of $200 \mathrm{kHz}$, and so ideally the transducer response would be flat across this frequency range. This particular transducer was designed to cover a wide bandwidth, having a polished flat backplate with a thin and light membrane. Furthermore, this design of transducer operated with uniform phase displacement across the aperture, producing a well ordered and easily simulated acoustic field. It was possible to improve and flatten the response at the lower frequency range (such as that used by $R$. aegyptiacus) through the use of a randomly rough backplate. However, this was at the expense of both phase response and highfrequency output. The device characterized and used here, allied with a spectral compensation technique provided the capability to reproduce signals across the entire frequency range of interest. This process is detailed in the following section.
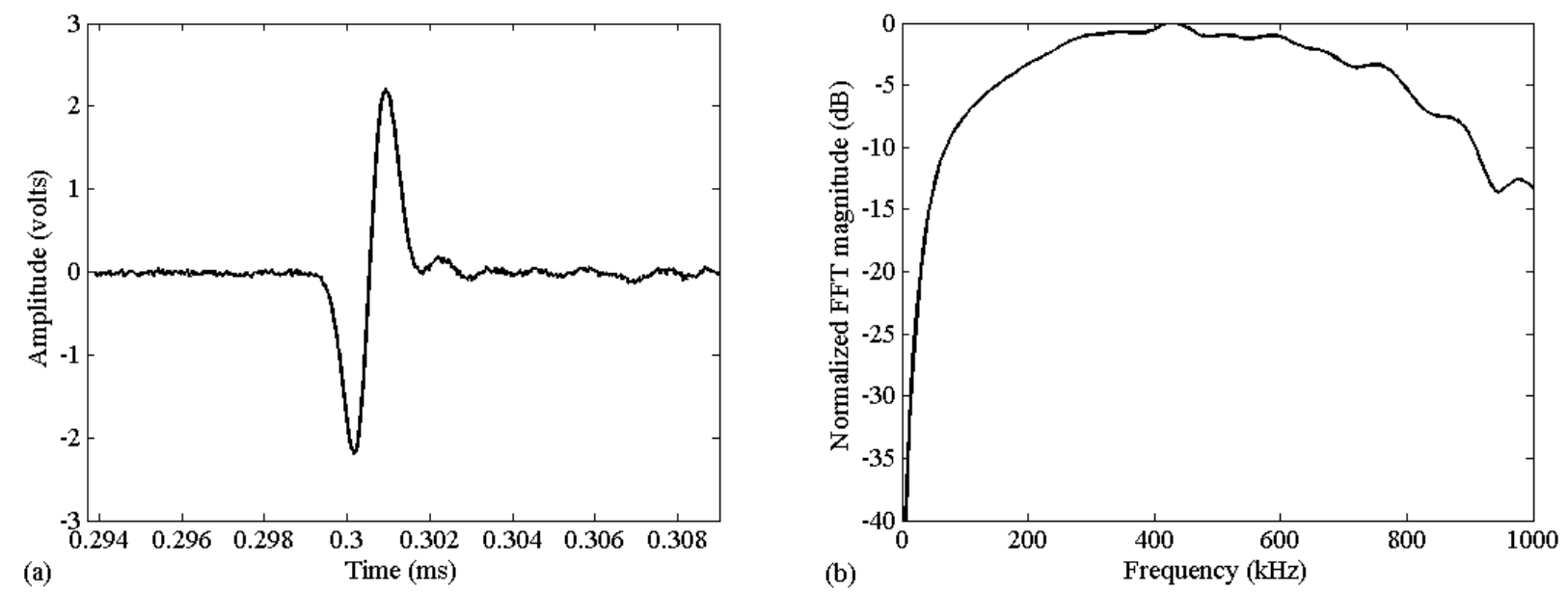

Figure 6. Transducer response measured in pitch-catch configuration at a separation of $100 \mathrm{~mm}$ : (a) impulse response; (b) FFT of impulse response illustrating frequency response.

\subsection{Recreating echolocation calls through use of a spectral processing technique}

The echolocation calls of $R$. aegyptiacus as recorded with the wireless sensor were reproduced in the laboratory using the wideband electrostatic transducer. The signal to be reproduced was taken from those signals recorded with the sensor in fixed-gain mode, providing the best SNR performance, as displayed in figure 4. The transducer output was recorded using the B\&K microphone, which has a flat response within $\pm 1 \mathrm{~dB}$ across the frequency range of the calls of $R$. aegyptiacus. The frequency response of the electrostatic transducer, however, 
was not flat across this frequency range, having a high-pass characteristic as depicted in figure 6(b). A method to equalize this response is detailed here, that allowed the input signal to be pre-processed to compensate for the system response.

Through laboratory measurements, it was shown that the response of the transducer was linear across the frequency range. Given this linearity, the response of the system is fully described by its impulse response, such that the output, $y(t)$, is defined as the convolution of the input, $x(t)$, with the impulse response, $h(t)$ :

$$
y(t)=h(t) * x(t)
$$

This calculation can be simplified by transforming into the frequency domain using a Fast Fourier Transform (FFT), where convolution of two signals is calculated by multiplication. Hence the output, $\mathrm{Y}(\mathrm{f})$, is the product of the input, $\mathrm{X}(\mathrm{f})$, and the impulse response, $\mathrm{H}(\mathrm{f})$, where all terms are complex:

$$
Y(f)=H(f) X(f)
$$

If a desired output signal is defined and the impulse response of the system is measured, then the input signal necessary to generate the desired output can be calculated, by rearrangement of (2):

$$
X(f)=\frac{Y(f)}{H(f)}
$$

Now, by transforming back to the time domain using the inverse FFT, the input signal required to generate the desired output can be found:

$$
x(t)=i F F T\left(\frac{Y(f)}{H(f)}\right)
$$

Hence, by measuring the impulse response of the system used to produce a signal, the input signal required to generate a defined output signal from the system can be calculated. This method produces a pre-distorted version of the required signal that, when used as the input signal, compensates for the impulse response of the system and hence allows accurate reproduction of a given signal.

The impulse response of the system was found by driving the electrostatic transducer with the Panametrics 5052 pulser unit, and capturing the output from the B\&K microphone. The desired output was defined as the $R$. aegyptiacus call recorded from the wireless sensor system. Using a Matlab (The Mathworks, MA, USA) script, the required drive signal was calculated from (4) and downloaded to the function generator to drive the transducer. The resulting transmitted signal is depicted in figure 7(a) where it can be seen that the signal reproduced using the inversion process shows good correlation with the original recording in the time domain. The need for the inversion process is demonstrated by making a comparison with the signal transmitted by the transducer using an unmodified input signal. This result is illustrated in figure 7(b), in the frequency domain for clarity. It can be seen that, as expected, the high-pass nature of the transducer response promotes the higher frequency components, which is compensated by the inversion process. 

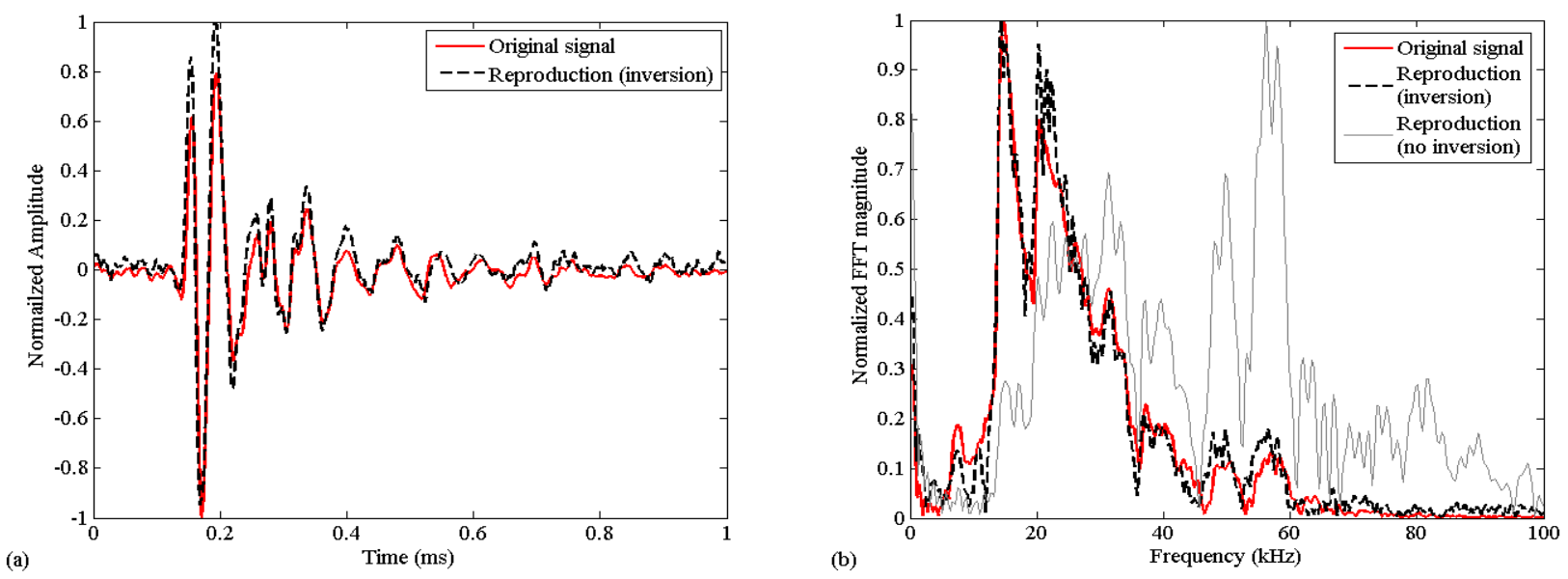

Figure 7. Reproduction of R. aegyptiacus echolocation call, comparing the original with a reproduction based on the inversion process in the time domain (a) and frequency domain (b). Also included in (b) is the frequency spectrum of a signal reproduced without use of the inversion process, applying the recorded echolocation call directly to the transducer.

The same procedure can be used to generate the more complex coded forms of bat call, such as FM calls, or those that contain a mixture of both FM and CF components (FM/CF). In this case, a biologically inspired, multi-harmonic FM chirp was created using Matlab. The signal consisted of a fundamental component (a quadratic chirp varying from $60 \mathrm{kHz}$ to $30 \mathrm{kHz}$ over $2.5 \mathrm{~ms}$ ) with four additional harmonics at amplitudes of $0.85,0.7,0.55$ and 0.4 relative to the fundamental component. This signal was designed to cover the entire frequency range of known bat echolocation calls. Its spectrogram is shown in figure 8(a) with the amplitude scale in dB. Figure $8(\mathrm{~b})$ shows the signal reproduced in the laboratory, using the same method as described previously and recorded using the B\&K microphone. It can be seen that agreement between the recorded and reproduced signal is again excellent. Figure $8(\mathrm{c})$ is displayed to illustrate the signal produced without using the inversion process. With this wideband signal, the inversion process compensated for the response of both the transducer and the microphone. In figure 8(c) it can be seen that the low-frequency roll-off of the transducer is responsible for a reduction in the relative amplitude of the fundamental component, while the reduction in sensitivity of the microphone above $100 \mathrm{kHz}$ causes the higher frequency components to be reduced in amplitude. Both these effects are effectively compensated.
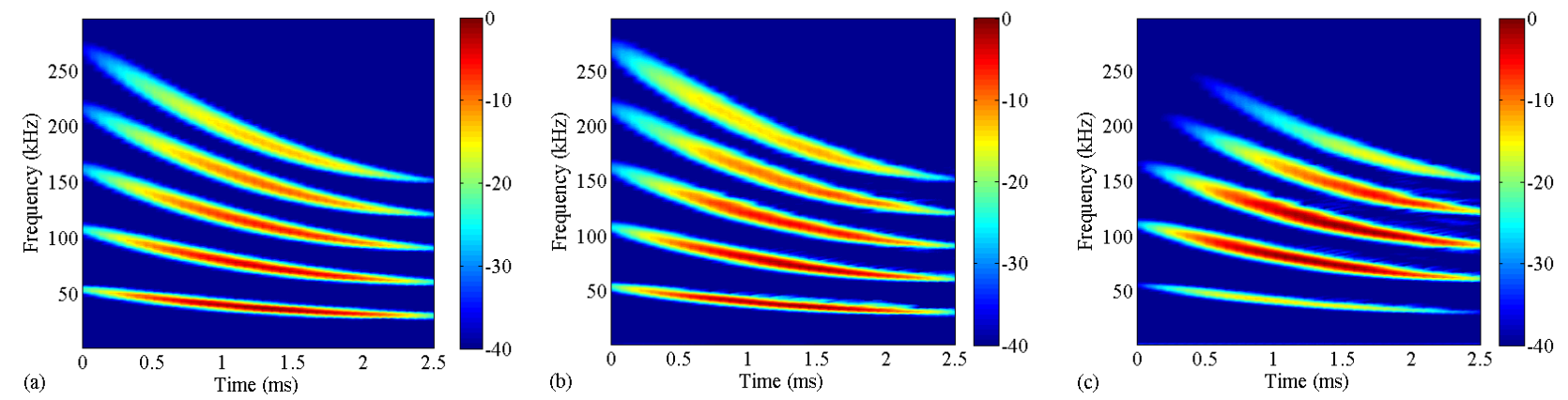

Figure 8. Reproduction of an artificial multi-harmonic FM chirp, shown in time-frequency (spectrogram) format. The original signal (a) and the signal reproduced using spectral inversion (b) show a high level of correlation. Also shown, is the signal reproduced without use of the inversion process (c). The scale is in $\mathrm{dB}$.

\section{Discussion and applications}

$R$. aegyptiacus is a member of the only megachiropteran genus of bat that echolocates. In this study a wireless sensor was designed to enable both emitted and reflected echolocation calls to be recorded whilst the bat was in flight. The emitted signals were then recreated in the laboratory using wideband custom-built electrostatic transducers, allied with a spectral equalization technique. This process was also demonstrated to be capable of reproducing a wideband $\mathrm{FM}$ signal, with frequency components ranging from $30 \mathrm{kHz}-300 \mathrm{kHz}$. 
By taking inspiration from the way in which bats perform gain control on their calls, the sensor was capable of operating over a dynamic range of greater than $70 \mathrm{~dB}$, including the static dynamic range of the system. This allowed both emitted and reflected echolocation calls to be recorded with the sensor in variable gain mode. In static gain mode, accurate recording of the emitted signal was promoted, whilst variable gain mode was used to obtain recordings of the echo signals returning to the bat. Through accurate calibration of the sensor system, its effect on the recorded signals can also be understood. With the signals emitted by $R$. aegyptiacus having a peak frequency of approximately $20 \mathrm{kHz}$, and little energy above $40 \mathrm{kHz}$, the sensor contributes an error of less than $\pm 5 \mathrm{~dB}$ across the range of these signals, demonstrating the importance of accurate calibration. The reflected signals detected by the variable gain sensor give some understanding of how complex the task of signal interpretation is, even in the relatively simple environment of a corridor. The images displayed here were specifically selected to contain distinct echoes; many of the sequences recorded had few, if any, distinct echoes. Rather, they generally contained a complex combination of overlapping reflected signals, which the bat must be able to decipher to obtain an image of the environment in which it is flying. It should be pointed out however, that while these signals were recorded returning to the bat, it does not necessarily hear them all. This is because, unlike the sensor, the bat has pinnae that it is known to actively scan (Holland and Waters, 2005) whilst echolocating. This may provide directional filtering of the returning echoes not available to the sensor microphone. Furthermore, directionality may be encoded into the echoes via the binaural head related transfer function (HRTF) of the bat, as is the case with other species (e.g. Aytekin et al., 2004). Techniques such as these would certainly appear to be required in order for the bat to obtain accurate spatial information from the plethora of reflected signals that are potentially available.

Reproduction of the echolocation calls of $R$. aegyptiacus was achieved using a purpose-built wideband electrostatic transducer allied to a spectral signal processing technique that compensated for the impulse response of the transducer system. The original aim of this work was to enable the generation of the complex, and often multiharmonic, calls of FM and CF-FM bats across a wide frequency range extending from $20 \mathrm{kHz}$ to in excess of $200 \mathrm{kHz}$. As such, the replication of R. aegyptiacus calls demonstrated the system working at the limit of its capabilities in terms of low frequency operation. Further testing was completed to show that the system and procedure was indeed capable of accurate reproduction of multiharmonic FM chirps, with frequency components up to $300 \mathrm{kHz}$. The success of this technique was further validation of the linearity of the transducer over a wide frequency range, and the flexibility of the spectral inversion technique. It should be noted that successful use of correlation-based methods requires that the received signal is an accurate reproduction of the transmitted signal. This constraint can be met through use of the spectral inversion process, since it takes the response of the entire system into account.

Accurate replication of bat calls opens up a variety of opportunities for future work, including validation of air propagation models with real echolocation signals or measurement of the target strength of prey insects (again with real echolocation signals). Biologically inspired signals could also be applied to engineering applications in different propagation media, such as improved target ranging and resolution in the underwater sonar environment and improved resolution for materials characterisation in solids and aggregate materials. A specific application of improved ranging through such wideband techniques is in robotic vehicle positioning systems for both air and underwater applications. In contrast to previous work employing single pulse excitation (e.g. Kuc, 1997) the application of wideband correlation-based detection offers improved resolution, but demands higher fidelity of signal generation. This is made possible through application of the spectral inversion process.

Finally, both sections of the paper have highlighted deficiencies in state of the art transducer technology for transmission or reception of airborne ultrasound over the frequency range of interest. The distinct null in the response of the Knowles microphone is problematic for accurate signal quantification across the range of frequencies used by bats, while the lower-frequency limitations of wideband electrostatic transducers required the use of pre-filtering for replication of calls covering this frequency range. In both cases, wider bandwidth transducers with greater sensitivity are required. This is the subject of current research by the authors and will be reported at a later date. 


\section{Acknowledgements}

The authors would like to acknowledge funding of the Biologically Inspired Acoustic Systems (BIAS) project from the Basic Technology Research Programme of the Research Councils UK, administered by EPSRC.

\section{References}

Aldridge, H.D.J.N. and Brigham, R.M. 1988 Load carrying and maneuverability in an insectivorous bat: a test of the 5\% rule of radio-telemetry. J. Mammal., 69(2), 379-82

Aytekin, M., Grassi, E., Sahota, M., and Moss, C. 2004 The bat head-related transfer function reveals binaural clues for sound localization in azimuth and elevation, J. Acoust. Soc. Am. 116(6), 3594-605

Fenton, M.B. and Bell, G.P. 1981. Recognition of species of insectivorous bats by their echolocation calls. $J$. Mammal. 62, 233-43

Friedrich, M., Dobie, G., Chan, C.C., Pierce, S.G., Galbraith, W., Marshall, S. and Hayward, G. 2009 Miniature mobile sensor platforms for condition monitoring of structures. IEEE Sens. J. 9(11), 1439 - 48

Ghose, K. and Moss, C.F. 2003. The sonar beam pattern of a flying bat as tracks tethered insects. J. Acoust. Soc. Am. 114(2), 1120-31

Griffin, D.R. 1944 How bats guide their flight by supersonic echoes. Am. J. Phys. 12, 342-5

Hartley, D.J. and Suthers, R.A. 1987 The sound emission pattern and the acoustical role of the noseleaf in the echolocating bat, Carollia perspicillata. J. Acoust. Soc. Am. 82(6), 1892-900

Henson Jr., O.W. 1965. The activity and function of the middle ear muscles in echolocating bats. J. Physiol. (London) 180, 871-87

Henson Jr., O.W., Bishop, A., Keating, A., Kobler, J., Henson, M., Wilson, B. and Hansen, R. 1987 Biosonar imaging of insects by Pteronotus p. parnellii, the mustached bat. Nat. Geog. Res. 3(1), 82-101

Hiryu, S., Katsura, K., Lin, L.-K., Riquimaroux, H. and Watanabe, Y. 2005 Doppler-shift compensation in the Taiwanese leaf-nosed bat (Hipposideros terasensis) recorded with a telemetry microphone system during flight. J. Acoust. Soc. Am. 118(6), 3927-33

Hiryu, S., Hagino, T., Riquimaroux, H. and Watanabe, Y. 2007 Echo-intensity compensation in echolocating bats (Pipistrellus abramus) during flight measured by a telemetry microphone. J. Acoust. Soc. Am. 121(3), 1749-57

Hiryu, S., Shiori, Y., Hosokawa, T., Riquimaroux, H. and Watanabe, Y. 2008 On-board telemetry of emitted sounds from free-flying bats: compensation for velocity and distance stabilises echo frequency and amplitude. J. Comp. Physiol. A 194, 841-51

Holderied, M.W., Korine, C., Fenton, M., Parsons, S., Robson, S. and Jones, G. 2005 Echolocation call intensity in the aerial hawking bat Eptesicus bottae (Vespertilionidae) studied using stereo videogrammetry. J. Exp. Biol. 208, 1321-7

Holland, R.A. and Waters, D.A. 2005 Echolocation signals and pinnae movement in the fruitbat Rousettus aegyptiacus. Acta Chiropterologica 7(1), 83-90.

Jen, P.H.-S. and Suga, N. 1975 Coordinated activities of the middle-ear and laryngeal muscles in echolocating bats. Science 191, 950-2

Kelly, S.P., Hayward, G. and Alvarez-Arenas, T.E.G. 2004 Characterization and assessment of an integrated matching layer for air-coupled ultrasonic applications. IEEE T. Ultrason. Ferr. 51(10) 1314-23

Kick, S.A. and Simmons, J.A. 1984 Automatic gain control in the bat's sonar receiver and the neuroethology of echolocation. J. Neurosci. 11(4), 2725-37

Koay, G., Heffner, R.S., Bitter, K.S. and Heffner, H.E. 2003 Hearing in leaf-nosed bats II: Carollia perspicillata. Hear. Res. 178, 27-34

Koay, G., Heffner, R.S. and Heffner, H.E. 1998 Hearing in a megachiropteran fruit bat (Rousettus aegyptiacus). J. Comp. Psychol. 112(4), 371-82

Korine, C., Speakman, J., Arad, Z. 2004 Reproductive energetics of captive and free-ranging Egyptian Fruit Bats (Rousettus aegyptiacus). Ecology 85(1), 220-30

Kuc, R. 1997 Biomimetic sonar recognizes objects using binaural information. J. Acoust. Soc. Am. 102(2), 68996

Lancaster, W.C., Keating, A.W. and Henson, O.W. 1992 Ultrasonic vocalizations of flying bats monitored by radiotelemetry. J. Exp. Biol. 173, 43-58 
Lancaster, W.C., Henson, O.W. and Keating, A.W. 1995 Respiratory muscle activity in relation to vocalization in flying bats. J. Exp. Biol. 198, 175-91

Lawrence B.D. and Simmons, J.A. 1982 Echolocation in bats: The external ear and perception of vertical targets. Science 218, 481-3

Manthey, W., Kroemer, N. and Magori, V. 1992 Ultrasonic transducers and transducer arrays for applications in air. Meas. Sci. Tech. 3, 249-61

Moss, C.F. and Schnitzler, H.-U. 1989 Accuracy of target ranging in echolocating bats: acoustic information processing. J. Comp. Physiol. A 165, 383-93

Neuweiler, G., Singh, S. and Sripathi, K. 1984 Audiograms of a South Indian bat community. J. Comp. Physiol. A 154, 133-42

Rafiq, M. and Wykes, C. 1991 The performance of capacitive ultrasonic transducers using v-grooved backplates. Meas. Sci. Technol. 2, 168-74

Schindel, D.W., Hutchins, D.A., Zou, L. and Sayer, M. 1995 The design and characterization of micromachined air-coupled capacitive transducers. IEEE T. Ultrason. Ferr. 42(1), 42-50

Simmons, J.A. 1973 The resolution of target range by echolocating bats. J. Acoust. Soc. Am. 54(1), 157-173

Simmons, J.A. 1979 Perception of echo phase information in bat sonar. Science 204, 1336-8

Simmons, J.A., Kick, S.A., Lawrence, B.D., Hale, C., Bard, C. and Escudie, B. 1983 Acuity of horizontal angle discrimination by the echolocating bat, Eptisicus fuscus. J. Comp. Physiol. A 153, 321-30

Simmons, J.A., Ferragamo, M., Moss, C.F., Stevenson, S.B. and Altes, A. 1990 Discrimination of jittered sonar echoes by the echolocating bat, Eptesicus fuscus: The shape of target images in echolocation. J. Comp. Physiol. A 167, 589-616

Simmons, J.A., Moffat, A.J., and Masters, W.M. 1992 Sonar gain control and echo detection thresholds in the echolocating bat, Eptesicus fuscus. J. Acoust. Soc. Am. 91, 1150-63.

Speakman, J.R. and Racey, P.A. 1991 No cost of echolocation for bats in flight. Nature 350, 421-3

Surlykke, A. and Kalko, E.K.V. 2008 Echolocating bats cry out loud to detect their prey. PLoS ONE, 3(4), e2036

Waters, D.A. and Jones, G. 1995 Echolocation call structure and intensity in five species of insectivorous bats. J. Exp. Biol. 198, 475-89

Waters, D.A. and Vollrath, C. 2003 Echolocation performance and call structure in the megachiropteran fruitbat Rousettus aegyptiacus. Acta Chiropterologica 5(2), 209-19 